\title{
Comparison of Direct Surgical Costs for Proximal Row Carpectomy and Four-Corner Arthrodesis
}

\author{
Nikolas H. Kazmers, MD, MSE ${ }^{1}$ Andrew R. Stephens, BS ${ }^{2}$ Angela P. Presson, $\mathrm{PhD}^{3,4}$ Yizhe $\mathrm{Xu}, \mathrm{MS}^{3,4}$ \\ Ross J. Feller, $M^{1} \quad$ Andrew R. Tyser, $M^{1}{ }^{1}$
}

\footnotetext{
1 Department of Orthopaedics, University of Utah, Salt Lake City, Utah

2 School of Medicine, University of Utah, Salt Lake City, Utah

${ }^{3}$ Division of Public Health, University of Utah, Salt Lake City, Utah

${ }^{4}$ Department of Pediatric Research Enterprise, University of Utah, Salt Lake City, Utah
}

J Wrist Surg 2019;8:66-71.
Address for correspondence Nikolas $\mathrm{H}$. Kazmers, MD, MSE, Department of Orthopaedics, University of Utah, 590 Wakara Way, Salt Lake City, UT 84108 (e-mail: nikolas.kazmers@utah.edu).

\author{
Abstract \\ Keywords \\ - cost \\ - four-corner \\ arthrodesis/fusion \\ - proximal row \\ carpectomy \\ - scaphoidectomy \\ - scapholunate \\ advanced collapse \\ wrist \\ - scaphoid nonunion \\ advanced collapse \\ wrist \\ - surgical encounter \\ - value
}

Background Proximal row carpectomy (PRC) and four-corner arthrodesis (FCA) are common treatments for stage II scapholunate advanced collapse (SLAC) and scaphoid nonunion advanced collapse (SNAC) wrists, with similar functional and patientreported outcomes reported in the peer-reviewed literature.

Questions Study questions included ( 1 ) whether surgical encounter total direct costs (SETDCs) differ between PRC and FCA, and (2) whether SETDC differs by method of fixation for FCA.

Patients and Methods Consecutive adult patients ( $\geq 18$ years) undergoing PRC and FCA between July 2011 and May 2017 at a single tertiary care academic institution were identified. Patients undergoing additional simultaneous procedures were excluded. Using our institution's information technology value tools, we extracted prospectively collected cost data for each surgical encounter. SETDCs were compared between PRC and FCA, and between FCA subgroups (screws, plating, or staples).

Results Of 42 included patients, mean age was similar between the 23 PRC and 19 FCA patients ( 51.2 vs. 54.5 years, respectively). SETDCs were significantly greater for FCA than PRC by $425 \%$. FCA involved significantly greater facility costs (2.3-fold), supply costs (10fold), and operative time (121 vs. 57 minutes). Implant costs were absent for PRC, which were responsible for $55 \%$ of the SETDC for FCA. Compared with compression screws, plating and staple fixation were significantly more costly (70\% and $240 \%$ greater, respectively). Conclusion SETDCs were $425 \%$ greater for FCA than PRC. Implant costs for FCA alone were $130 \%$ greater than the entire surgical encounter for PRC. For FCA, SETDC varied depending on the method of fixation.

Level of Evidence This is a level III, cost analysis study.
The cost of health care delivered in the United States has received increased attention in the past decade, in part due to policy changes that have included value-based payment models. ${ }^{1,2}$ Proximal row carpectomy (PRC) and four-corner arthrodesis (FCA) are common surgical procedures for stage I-II scapholunate advanced collapse (SLAC) and scaphoid nonunion advanced collapse (SNAC) patterns of wrist arthritis. 3,4 Substantial efforts have previously been made to assess clinical outcomes following PRC and FCA, ${ }^{5-22}$ although prior work elucidating cost differences is lacking. Such information may received

March 15, 2018

accepted after revision

September 24, 2018

published online

November 16, 2018
Copyright $\odot 2019$ by Thieme Medical

Publishers, Inc., 333 Seventh Avenue, New York, NY 10001, USA Tel: +1(212) 584-4662.
DOI https://doi.org/ 10.1055/s-0038-1675791. ISSN 2163-3916. 
help guide treatment of patients with stage I-II SLAC or stage I SNAC wrist, as the treating surgeon may have the option to perform either procedure.

The findings of numerous single-center investigations into clinical outcomes following PRC and FCA have been conglomerated by two systematic reviews. ${ }^{5,6}$ PRC, as compared with FCA, was associated with a 6- to 11-degree greater arc of wrist flexion-extension, and fewer complications were reported. However, no significant differences were observed in Disabilities of the Arm, Shoulder and Hand (DASH) scores, rate of conversion to total wrist arthrodesis (TWA), increase in grip strength in proportion to the contralateral side, pain relief, or subjective outcomes on a Likert scale. ${ }^{5,6}$

Given similar clinical outcomes reported in the peerreviewed literature for PRC and FCA, the discussion related to what constitutes appropriate treatment should also focus on improving value through cost reduction in addition the prior focus upon patient age, labor status, smoking status, and sex when choosing the treatment technique. ${ }^{16-18,23}$ Current literature related to the economic and cost aspects of PRC and FCA is limited. ${ }^{24}$

Our institution has developed a "Value Driven Outcomes" (VDO) database containing detailed patient- and item-level total direct cost and payment data for a variety of health care services. This has successfully identified areas of high variability in cost, leading to improved value of care delivered. ${ }^{1,25,26}$ In this study, the VDO tool was utilized to answer our primary study question of whether surgical costs differ between PRC and FCA. Similarly, our second study question, whether surgical costs differ based on the type of fixation used for FCA (headless compression screws, plate/screw construct, or staples), was investigated.

\section{Patients and Methods}

This Institutional Review Board-approved retrospective cost analysis study included all consecutive adult ( $\geq 18$ years of age) patients undergoing isolated PRC or FCA between July 2011 and May 2017 performed by four fellowshiptrained hand surgeons at a single tertiary academic institution. Patients were identified by Current Procedural Terminology (CPT) code (PRC 25215; FCA 25820 and 25825), and corresponding basic demographic and surgical data were tabulated. Manual chart review of all operative, anesthesia, and orthopaedic clinic notes was performed to record the diagnosis, surgical procedures performed, use of bone graft and fixation type used for FCA, and anesthesia type. Patients undergoing additional simultaneous procedures including other surgeries or injections, and those treated for a primary diagnosis of infection (i.e., neglected wrist septic arthritis with carpal osteomyelitis), were excluded. Also excluded were patients undergoing revision PRC or FCA as these cases are not representative of primary surgeries and would be expected to cost more, and those treated prior to July 2011 (corresponding with initiation of the VDO database at our institution). We did not exclude patients diagnosed with complications subsequent to the index procedure, and we did not adjust costs of the index procedure to include costs related to the treatment of subsequent complications.
PRC and FCA were performed as previously described through longitudinal dorsal wrist excisions. ${ }^{7}$ All surgeries were performed under general anesthesia, or under a surgical block with intravenous sedation, according to patient preference. Longitudinal or ligament sparing capsulotomies were performed based on the surgeon's preference. PRC patients did not receive capsular or allograft interposition. FCA fixation included headless compression screws, circular locking plate and screw constructs, or compressive staples based on the surgeon's preference. Bone autograft from the distal radius and/or excised scaphoid was used for all FCA cases. All patients were placed in a plaster forearm-based splint in the operating room.

Patients were indicated to undergo FCA if midcarpal arthrosis was observed intraoperatively (stage III SLAC or stage II SNAC patients). Those with radiolunate involvement treated with TWA were excluded from the study. In the setting of stage I-II SLAC or stage I SNAC, the choice of PRC and FCA was determined through a shared decision-making discussion between the surgeon and patient while considering patient age, functional demands, smoking status, and medical comorbidities. Although both procedures have yielded similar clinical outcomes in the literature, ${ }^{5,27}$ we were more likely to recommend PRC for smokers and older patients with multiple comorbidities or low functional demands, and FCA for young, active, nonsmokers.

Total direct cost and payment data were collected from the VDO database for each individual surgery performed. The VDO information technology tool draws prospectively collected payment data and patient- and item-level total direct cost data from our institution's data warehouse for specific patient encounters. VDO costing methods have been previously described, yielding total direct costs for materials used for patient care, facility utilization direct costs (including sterile processing costs), and time-based cost allocations including procedure/operative time and cost of staff involved in care (nursing, surgical technicians, medical assistants). 1,25,26 Total direct cost categories, as tabulated by the VDO tool, are further described in - Appendix A. All reported cost data were normalized using each individual's cost divided by the average cost in the data set, to comply with institutional guidelines prohibiting the public reporting of any financial data related to the details of nonpublically disclosed contractual agreements, or raw cost data.

Continuous variables were summarized as mean and standard deviation (SD), and categorical variables were summarized as count and percentage (\%). Relative costs were calculated relative to the lowest group by dividing each distinct group average by the lowest group mean. Total direct costs were compared between PRC and FCA groups using a Wilcoxon rank sum test. The Kruskal-Wallis test was used to compare total direct costs between FCA subgroups based on the method of fixation (screws, plating, or staples), followed by Tukey's adjustment for multiple pairwise comparisons. Age was compared using the Student's t-test, and categorical demographic variables were compared using the Fisher's exact test. Statistical significance was assessed at the 0.05 level, and all applicable tests were two-tailed. 
Table 1 Baseline patient characteristics

\begin{tabular}{|c|c|c|c|}
\hline Factor & $\begin{array}{l}\text { PRC } \\
(n=23)\end{array}$ & $\begin{array}{l}\text { FCA } \\
(n=19)\end{array}$ & $p$-Value \\
\hline Age $(y \pm S D)$ & $54.5 \pm 9.8$ & $51.2 \pm 14.7$ & 0.35 \\
\hline Sex (female) ${ }^{a}$ & $12(52 \%)$ & $3(16 \%)$ & $<0.05$ \\
\hline Insurance type ${ }^{a}$ & & & 0.68 \\
\hline Commercial & $15(65 \%)$ & $13(68 \%)$ & - \\
\hline Medicare & $4(17 \%)$ & $5(26 \%)$ & - \\
\hline Medicaid & $2(9 \%)$ & $1(5 \%)$ & - \\
\hline Other & $2(9 \%)$ & $0(0 \%)$ & - \\
\hline Diagnosis $^{a}$ & & & 0.08 \\
\hline SLAC wrist & 7 (30\%) & $12(63 \%)$ & - \\
\hline SNAC wrist & $14(61 \%)$ & $7(37 \%)$ & - \\
\hline Kienbock's disease & $2(9 \%)$ & $0(0 \%)$ & - \\
\hline SLAC/SNAC stage ${ }^{a}$ & & & $<0.05$ \\
\hline$|-| \mid$ & $21(91 \%)$ & $9(39 \%)$ & - \\
\hline III & $0(0 \%)$ & $10(61 \%)$ & - \\
\hline IV & $0(0 \%)$ & $0(0 \%)$ & - \\
\hline
\end{tabular}

Abbreviations: FCA, four-corner arthrodesis; PRC, proximal row carpectomy; SD, standard deviation; SLAC, scapholunate advanced collapse; SNAC, scaphoid nonunion advanced collapse.

Note: Continuous data presented as mean \pm standard deviation; categorical data presented as number of patients and (percentage). aBased on Fisher's exact test contingency tables.

We based our sample size calculations on an effect size of 1.43 in SD units. We obtained a 1:4.3 ratio of PRC group versus FCA group based on our analyses. With a two-sided, twosample $t$-test, and with a total sample size of 42 (19 FCA and 23 PRC), we have $99 \%$ power to detect a medium effect size of 1.43 for total direct cost between groups at a $0.05 \alpha$ level.

\section{Results}

Of 41 patients identified that underwent PRC, 18 were excluded: 11 underwent additional surgical procedures, 5 carried a diagnosis of infection, and 2 were revision surgeries. Of 31 identified FCA patients, 12 were excluded: 9 underwent additional simultaneous surgical procedures, and 3 were revisions. Therefore, a total of 42 patients (23 PRC, 19 FCA) were included for final analysis.

Baseline patient characteristics are illustrated in - Table 1. Mean age was similar between PRC and FCA groups (51.2 vs. 54.5 years, respectively; $p=0.35$ ). However, the PRC group included more females (52\% vs. $16 \%$, respectively; $p<0.05$ ) and a lower proportion of stage III SLAC/SNAC wrist. Payer mix was similar between groups. No patient undergoing PRC received implantable hardware (including temporary Kirschner wires), and all patients undergoing FCA received bone autograft. There were no differences in the use of general anesthesia between groups (PRC 78\% general and 22\% surgical block with sedation; FCA $100 \%$ general anesthetic; $p=0.82$ ).

Surgical encounter total direct costs (SETDCs) were $425 \%$ greater for FCA than PRC $(p<0.05)$, which is illustrated in - Fig. 1 (relative areas of PRC and FCA pie charts are proportional). FCA was associated with significantly greater facility utilization costs (2.3-fold; $p<0.05)$, supply costs (10-fold; $p<0.05$ ), and operative time (121 vs. 57 minutes; $p<0.05$ ). There were no differences in pharmacy costs $(p=0.09)$, or other services which include operating room and recovery room staff costs $(p=0.68)$. Implant costs comprised $55 \%$ of the SETDC for FCA, and were absent for PRC, which precludes formal statistical comparison between groups.

Three methods of fixation were observed among the FCA cohort: headless compression screws, circular plate and screw constructs, and staples. Surgical time did not differ between the three methods of fixation $(116,130$, and 116 minutes, respectively; $p=0.32$ ). For patients receiving headless compression

\section{Proximal Row Carpectomy (PRC)}
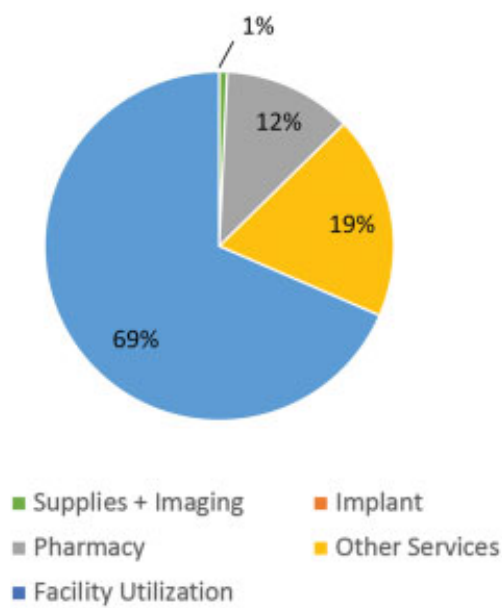

\section{Four Corner Arthrodesis (FCA)}

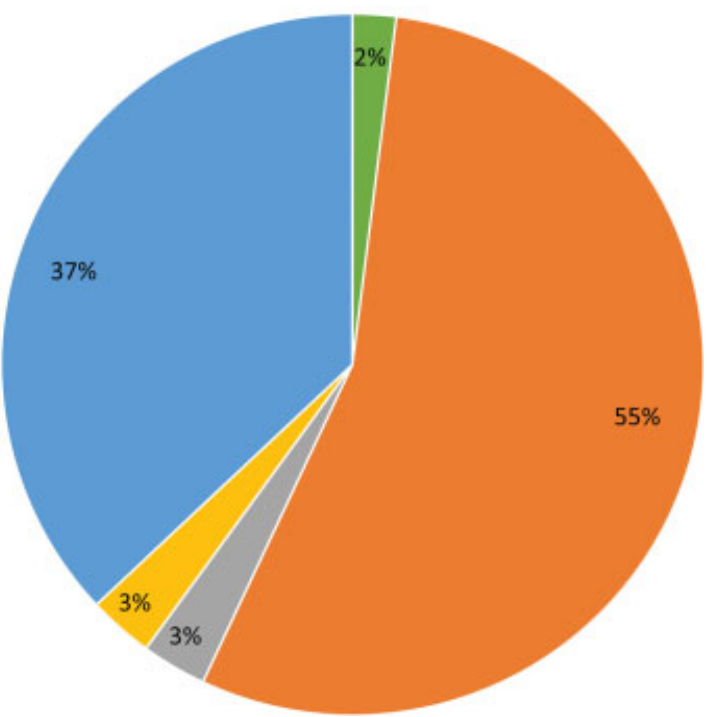

Fig. 1 Comparison of surgical encounter total direct costs for proximal row carpectomy (PRC) and four-corner arthrodesis (FCA). PRC was defined as the reference group (pie chart area normalized to 1.0). 


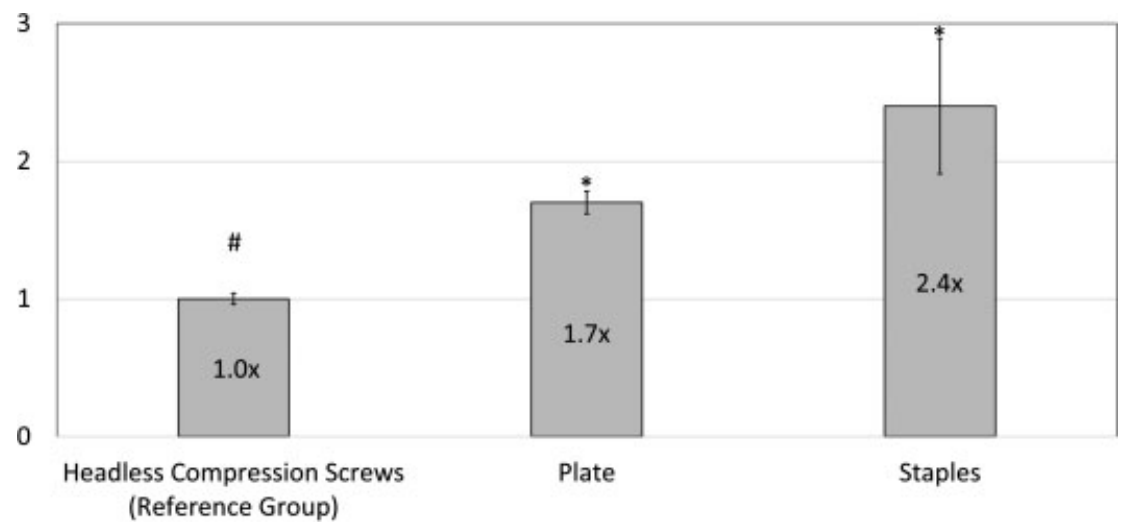

Fig. 2 Data represent mean \pm standard error of the mean (SEM). \#Reference group, normalized to 1.0. ${ }^{*} p<0.05$ as compared with left-sided neighboring value per Kruskal-Wallis tests and Tukey's multiple pairwise comparison tests. Values over graph bars represent fold-change differences relative to the reference group.

screw fixation, two screws were used in all cases. For those receiving staple fixation, an average of $1.9 \pm 1.0$ staples were used. - Fig. 2 illustrates that the SETDC for FCA differed significantly for each of these three fixation types $(p<0.05$ for the Kruskal-Wallis test; $p<0.05$ for Tukey's pairwise comparisons evaluating for differences in SETDC between plate/screws versus headless compression screws, and headless compression screws versus staples).

\section{Discussion}

Our main finding was that FCA direct surgical costs were 425\% greater than PRC. Responsible for this difference were implant costs which were absent for the PRC group and amounted to a value of $130 \%$ greater than the entire surgical encounter cost for PRC, facility costs in which the surgical time for FCA was approximately double of that for PRC, and supply costs which were 10 -fold greater for FCA and were attributable to disposable drill bits and burr tips unique to this group. Therefore, we rejected our null hypothesis that there are no cost differences between the two procedures.

Prior literature informing the clinical decision between PRC and FCA is limited. Although no power calculations were provided and the study was likely underpowered, a clinical trial comparing 13 PRC and 14 FCA patients with stage II SNAC arthritis found no significant differences in DASH or Visual Analog Scale pain scores with a follow-up of 45 to 73 months. ${ }^{15}$ This clinical scenario is of particular interest, as the treating surgeon may perform either procedure. Patient age, sex, smoking status, and labor status are also factors to consider when choosing treatment given the potential effects on the durability of PRC or the risk of nonunion following FCA. ${ }^{16-18,23}$ Additionally, the lower rate of complications associated with PRC may guide treatment choices., ${ }^{5,6}$

Graham and Detsky concluded that PRC was preferable over FCA and TWA based on a decision analysis study published in $2001 .^{28}$ Subsequently, similar clinical outcomes for both procedures have been reported, including similar levels of postoperative pain improvement, subjective and objective (DASH) outcomes improvement, and conversion to total wrist fusion. ${ }^{5,6}$ Although statistically significant, it is questionable whether a difference in flexion-extension of 6 to 11 degrees favoring PRC is clinically significant. ${ }^{5,6}$

Our findings regarding differences in surgical direct costs between PRC and FCA add to findings reported in the previous literature. Rahgozar et al recently evaluated cost differences between PRC and intercarpal arthrodesis using payment data from the MarketScan database, which is comprised of a convenience sample of commercially insured patients. ${ }^{24,29}$ The authors concluded that PRC was 33.9\% less expensive than intercarpal arthrodesis, as calculated from published payment estimates. Intercarpal arthrodesis patients were assumed to have undergone FCA based on CPT and International Classification of Diseases, Ninth Revision (ICD-9) code searches for intercarpal arthrodesis and intercarpal osteoarthritis, respectively. However, without the ability to perform manual chart review or review of radiographs, combined with the nonspecificity of these CPT and ICD-9 codes, it was not possible to identify the type of intercarpal arthrodesis the included patients received. It is probable that some of these patients underwent scaphotrapeziotrapezoidal, scaphocapitate, or radioscapholunate arthrodeses which could be coded identically. Although this study contained a smaller sample size from a single institution, we were able to confirm the surgical treatments of all included patients, and exclude those undergoing additional simultaneous procedures which could affect costs. Although payment may serve as a surrogate for cost, the MarketScan database reports payment data rather than true direct costs, ${ }^{29,30}$ and the reported values were obtained through multivariable regression analysis that included payments related to the index procedure plus those related to follow-up procedures (hardware removal, further fusion surgeries). Therefore, our findings add to the findings of the prior study by demonstrating surgical direct costs differ between PRC and FCA. Due to the aforementioned limitations, the authors' conclusion that FCA was more likely to be converted to TWA than PRC should be interpreted with caution.

As a secondary finding, we observed that the method of fixation did not affect operative time, but did significantly affect surgical direct costs for the FCA group. At our institution, use of headless compression screws was the least expensive form of fixation in which two screws were used for all cases, 
with increasing costs associated with use of circular plate and screw constructs, and staples (mean of 1.9 used per case) as the most expensive construct. Although implant costs may differ at other institutions, surgeon awareness of potential cost differences between available constructs may provide an opportunity for cost reduction for FCA.

Limitations of this study deserve mention. Although each surgical procedure was verified with chart review, the initial identification of patients by procedure code, the retrospective study design, and evaluation of patients from a single center introduce potential for selection bias. Due to the nonrandomized study design, significant difference in sex between the PRC and FCA groups was observed. However, it is unclear if these differences would affect procedural costs. We included two patients undergoing PRC for Kienbock's disease, which technically is similar to performing the procedure for SLAC or SNAC wrist but may affect surgical costs. Generalizability of our results may be limited by our unique pricing agreements between our institution and suppliers, which may affect total direct costs of implants, time-allocated costs of perioperative services, and other products used during the surgical encounter. The ability to generalize our results to other reported surgical techniques including dorsal capsular interposition ${ }^{31}$ or osteochondral resurfacing of the capitate ${ }^{32}$ with PRC, three-corner arthrodesis, ${ }^{27}$ or FCA using only buried Kirschner wires for fixation is uncertain. Similarly, surgeons at other institutions may use different numbers of staples or headless compression screws when performing PRC, which could affect the generalizability of our results for the FCA subanalysis by fixation method. We did not quantify the opportunity cost for PRC versus FCA, in which the latter required twice the surgical time. The VDO database does not include indirect cost data such as housekeeping, electricity, or property rent/depreciation. Additionally, the study was not designed to evaluate costs related to time off of work related to patient recovery. In general, PRC and FCA patients were permitted to return to desk work within 3 days of surgery, and heavy labor was permitted around 10 weeks postoperatively for both groups-however, we are unable to comment on the exact duration of time off of work for this study population. Although this does not hinder the primary aim of the study, which is to compare direct surgical costs between PRC and FCA, including these indirect costs and those related to complications in an updated cost-effectiveness analysis study would be informative. ${ }^{28}$

In conclusion, we found that FCA direct surgical costs were 425\% greater than PRC. Implant costs for FCA were $130 \%$ greater than the entire surgical cost for PRC. In light of these findings and considering prior clinical literature describing outcomes for both procedures and risk factors for failure, we would suggest that the apparent cost difference between PRC and FCA is a relevant additional consideration when choosing the appropriate treatment for indicated patients.

\section{Ethical Approval}

This study was approved by our university's Institutional Review Board.

\section{Funding}

One or more of the authors (A.P.P.) has received funding from the National Institutes of Health $(\mathrm{NIH})$ in the form of a grant-this investigation was supported by the University of Utah Study Design and Biostatistics Center, with funding in part from the National Center for Research Resources and the National Center for Advancing Translational Sciences, NIH, through Grant 5UL1TR001067-02 (formerly 8UL1TR000105 and UL1RR025764).

\section{Conflict of Interest}

None.

\section{References}

1 Lee VS, Kawamoto K, Hess R, et al. Implementation of a value-driven outcomes program to identify high variability in clinical costs and outcomes and association with reduced cost and improved quality. JAMA 2016;316(10):1061-1072

2 Kawamoto K, Martin CJ, Williams K, et al. Value Driven Outcomes (VDO): a pragmatic, modular, and extensible software framework for understanding and improving health care costs and outcomes. J Am Med Inform Assoc 2015;22(01):223-235

3 Watson HK, Ballet FL. The SLAC wrist: scapholunate advanced collapse pattern of degenerative arthritis. J Hand Surg Am 1984;9 (03):358-365

4 Harrington RH, Lichtman DM, Brockmole DM. Common pathways of degenerative arthritis of the wrist. Hand Clin 1987;3(04): 507-527

5 Saltzman BM, Frank JM, Slikker W, Fernandez JJ, Cohen MS, Wysocki RW. Clinical outcomes of proximal row carpectomy versus fourcorner arthrodesis for post-traumatic wrist arthropathy: a systematic review. J Hand Surg Eur Vol 2015;40(05):450-457

6 Mulford JS, Ceulemans LJ, Nam D, Axelrod TS. Proximal row carpectomy vs four corner fusion for scapholunate (SLAC) or scaphoid nonunion advanced collapse (SNAC) wrists: a systematic review of outcomes. J Hand Surg Eur Vol 2009;34(02):256-263

7 Cohen MS, Kozin SH. Degenerative arthritis of the wrist: proximal row carpectomy versus scaphoid excision and four-corner arthrodesis. J Hand Surg Am 2001;26(01):94-104

8 Dacho AK, Baumeister S, Germann G, Sauerbier M. Comparison of proximal row carpectomy and midcarpal arthrodesis for the treatment of scaphoid nonunion advanced collapse (SNAC-wrist) and scapholunate advanced collapse (SLAC-wrist) in stage II. J Plast Reconstr Aesthet Surg 2008;61(10):1210-1218

9 DiDonna ML, Kiefhaber TR, Stern PJ. Proximal row carpectomy: study with a minimum of ten years of follow-up. J Bone Joint Surg Am 2004;86-A(11):2359-2365

10 Jebson PJ, Hayes EP, Engber WD. Proximal row carpectomy: a minimum 10-year follow-up study.J Hand Surg Am 2003;28(04):561-569

11 Kendall CB, Brown TR, Millon SJ, Rudisill LE Jr, Sanders JL, Tanner SL. Results of four-corner arthrodesis using dorsal circular plate fixation. J Hand Surg Am 2005;30(05):903-907

12 Traverso P, Wong A, Wollstein R, Carlson L, Ashmead D, Watson HK. Ten-year minimum follow-up of 4-corner fusion for SLAC and SNAC wrist. Hand (NY) 2017;12(06):568-572

13 Vanhove W, De Vil J, Van Seymortier P, Boone B, Verdonk R. Proximal row carpectomy versus four-corner arthrodesis as a treatment for SLAC (scapholunate advanced collapse) wrist. J Hand Surg Eur Vol 2008;33(02):118-125

14 Bain GI, Watts AC. The outcome of scaphoid excision and fourcorner arthrodesis for advanced carpal collapse at a minimum of ten years. J Hand Surg Am 2010;35(05):719-725

15 Aita MA, Nakano EK, Schaffhausser HL, Fukushima WY, Fujiki EN. Randomized clinical trial between proximal row carpectomy and 
the four-corner fusion for patients with stage II SNAC. Rev Bras Ortop 2016;51(05):574-582

16 Chim H, Moran SL. Long-term outcomes of proximal row carpectomy: a systematic review of the literature. J Wrist Surg 2012;1(02): 141-148

17 Wagner ER, Bravo D, Elhassan B, Moran SL. Factors associated with improved outcomes following proximal row carpectomy: a longterm outcome study of 144 patients. J Hand Surg Eur Vol 2016;41 (05):484-491

18 Wagner ER, Werthel JD, Elhassan BT, Moran SL. Proximal row carpectomy and 4-corner arthrodesis in patients younger than age 45 years. J Hand Surg Am 2017;42(06):428-435

19 Berkhout MJ, Bachour Y, Zheng KH, Mullender MG, Strackee SD, Ritt MJ. Four-corner arthrodesis versus proximal row carpectomy: A retrospective study with a mean follow-up of 17 years. J Hand Surg Am 2015;40(07):1349-1354

20 Richou J, Chuinard C, Moineau G, Hanouz N, Hu W, Le Nen D. Proximal row carpectomy: long-term results. Chir Main 2010;29 (01):10-15

21 Ferreres A, Garcia-Elias M, Plaza R. Long-term results of lunocapitate arthrodesis with scaphoid excision for SLAC and SNAC wrists. J Hand Surg Eur Vol 2009;34(05):603-608

22 Williams JB, Weiner $\mathrm{H}$, Tyser AR. Long-term outcome and secondary operations after proximal row carpectomy or four-corner arthrodesis. J Wrist Surg 2018;7(01):51-56

23 Wall LB, Didonna ML, Kiefhaber TR, Stern PJ. Proximal row carpectomy: minimum 20-year follow-up. J Hand Surg Am 2013;38(08): 1498-1504

\section{Appendix A: Breakdown of Value-Driven Outcomes Database Categories for Total Direct Costs}

IMAGING COST: All imaging, scans, and radiology services used.

SUPPLY COST: All supplies and devices used, excluding implants. This includes disposable drill bits, burr tips, and saw blades.

IMPLANT COST: All supply costs related to surgical implants.

PHARMACY COST: Total cost of all medication used during the patient encounter.

LAB COST: All lab work associated with the visit, including blood work, urinalysis, hematology, and all other lab or chemistry-related costs.
24 Rahgozar P, Zhong L, Chung KC. A comparative analysis of resource utilization between proximal row carpectomy and partial wrist fusion: a population study. J Hand Surg Am 2017;42(10):773-780

25 Chalmers PN, Granger E, Nelson R, Yoo M, Tashjian RZ. Factors affecting cost, outcomes, and tendon healing after arthroscopic rotator cuff repair. Arthroscopy 2018;34(05):1393-1400

26 Tashjian RZ, Belisle J, Baran S, et al. Factors influencing direct clinical costs of outpatient arthroscopic rotator cuff repair surgery. J Shoulder Elbow Surg 2018;27(02):237-241

27 Delattre O, Goulon G, Vogels J, Wavreille G, Lasnier A. Threecorner arthrodesis with scaphoid and triquetrum excision for wrist arthritis. J Hand Surg Am 2015;40(11):2176-2182

28 Graham B, Detsky AS. The application of decision analysis to the surgical treatment of early osteoarthritis of the wrist. J Bone Joint Surg Br 2001;83(05):650-654

29 Truven. MarketScan research databases; 2017. Available at: https:// truvenhealth.com/your-healthcare-focus/analytic-research/market scan-research-databases. Accessed December 12, 2017

30 Peterson C, Xu L, Florence C, et al. The medical cost of abusive head trauma in the United States. Pediatrics 2014;134(01):91-99

31 Kwon BC, Choi SJ, Shin J, Baek GH. Proximal row carpectomy with capsular interposition arthroplasty for advanced arthritis of the wrist. J Bone Joint Surg Br 2009;91(12):1601-1606

32 Tang P, Wei DH, Ueba H, Gardner TR, Rosenwasser MP. Scaphoid excision and 4-bone arthrodesis versus proximal row carpectomy: a comparison of contact biomechanics. J Hand Surg Am 2012;37(09): 1861-1867

OTHER SERVICES COST: Services that do not fall into one of the other categories. Services include Physical Therapy, Occupational Therapy, Speech Pathology, Respiratory Service, EKG, Recovery Room nursing/staff, and Other Therapeutic Services.

FACILITY UTILIZATION COST: Time and labor costs for patients stay in each unit (excluding Professional costing such as time-allocated estimates of physician costs). Cost is mapped to the individual patient level based on time spent on specific unit (surgery minutes, patient hours) or by completed visit. 